Kompass

Ophthalmologie

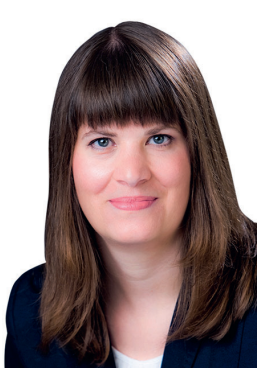

Stephanie Joachim

Koordination «OphthalmoCampus»

\title{
Was Ärzte bewegt
}

Liebe Kolleginnen und Kollegen,

vor fast drei Jahren durfte ich Sie an dieser Stelle zur ersten Ausgabe des «OphthalmoCampus» im Kompass OPHTHALMOLOGIE begrüßen. Jetzt ist es für mich an der Zeit die Koordination des Campus, der mir in dieser Zeit sehr ans Herz gewachsen ist, abzugeben. Und so freut es mich sehr, dass Frau Dr. med. Dipl. Mol. Med. Univ. Bettina Hohberger von der Universitäts-Augenklinik Erlangen den Campus in Zukunft betreuen wird.

Ich möchte mich bei Ihnen fürs Lesen und beim Lektorat des KOMPASs für die schöne und harmonische Zusammenarbeit zu bedanken! Und wünsche bei der Lektüre des aktuellen «OphthalmoCampus» viel Vergnügen und alles Gute für das neue Jahr! 


\section{Weiterbildung \\ Fachübergreifende Sekundäranalyse von Daten aus Befragungen zur Arbeits- und Weiterbildungs- situation junger Ärztinnen und Ärzte in Deutschland}

Das Bündnis Junge Ärzte (BJÄ), in dem viele Nachwuchsgruppen verschiedenster Fachbereiche organisiert sind, nutzte seine Reichweite, um den aktuellen und fachübergreifenden Status Quo der Weiterbildungs- und Arbeitsbedingungen junger Ärztinnen und Ärzte in Deutschland zu analysieren. Gerade Änderungen im Gesundheitssystem in den letzten Jahren hatten starken Einfluss auf den Arbeitsalltag der jungen Ärzteschaft. Für das Projekt des BJÄ wurden sich überschneidende Daten aus 6 Einzelbefragungen gemeinsam in Form einer Sekundäranalyse ausgewertet. Insgesamt gingen in die Analyse 4041 komplette Datensätze ein. Inhalt aller 6 Befragungen war neben wichtigen Themenfeldern wie den Arbeitsbedingungen im Berufsalltag und der Weiterbildung sowie der Vereinbarkeit von Beruf und Familie auch das Modell beruflicher Gratifikationskrisen zur Beurteilung psychosozialer Arbeitsbelastung.

Im Folgenden soll ein Überblick über zentrale Ergebnisse der Analyse gegeben werden: Es wurde deutlich, dass ein hoher Anteil der Arbeitszeit durch nicht unmittelbar ärztliche Aufgaben eingenommen wird. Durchschnittlich wurde allein ein Viertel der Arbeitszeit (25 $\pm 15 \%$; Mittelwert \pm Standardabweichung) auf patientenferne Tätigkeiten, wie Befunde und Termine organisieren oder Diagnosen kodieren, verwendet. Die Arbeit direkt am Patienten nahm durchschnittlich 44 $\pm 19 \%$ der Arbeitszeit ein, 32 $\pm 13 \%$ wurden auf patientenbezogene Tätigkeiten wie Fallbesprechungen und Schreiben von Arztbriefen verwendet. Die zunehmende Bürokratisierung und Reglementierung der Medizin in den letzten Jahren ist am ehesten als Grund dieses großen Anteils an nicht unmittelbar ärztlichen Aufgaben zu sehen. Die Mehrheit der Befragten sahen diese vielschichtigen Veränderungen im ärztlichen Arbeitsumfeld deutlich kritisch: Auf die Frage, ob die Qualität der Patientenversorgung dadurch gefährdet sei, antworteten 47\% der Befragten mit «Ja, eher schon» und $40 \%$ mit «Ja, sehr deutlich». Die Befragten wünschten mehrheitlich eine Reduktion der Arbeitsverdichtung z.B. durch eine Entlastung bei organisatorischen Aufgaben und der Dokumentation. Obwohl die aktuellen Arbeitsbedingungen kritisch bewertet wurden, war die allgemeine Zufriedenheit der Befragten mit ihrem Berufsalltag relativ hoch: 10\% der Teilnehmer gaben an «sehr zufrieden», 36\% «eher zufrieden», 28\% «unentschieden», 20\% «eher unzufrieden» und nur 6\% «sehr unzufrieden» zu sein. Nach Ansicht der Autoren lässt sich diese Diskrepanz am ehesten durch eine tiefgehende Identifikation der Ärzteschaft mit ihrem Beruf erklären.

Die psychosoziale Arbeitsbelastung war bei den Teilnehmern der Studie mit einer ER-Ratio von 1,8 stark ausgeprägt. Die ER-Ratio ergibt sich aus 16 Items und setzt Belastungsfaktoren (engl. effort) und Belohnungsfaktoren (engl. reward) ins Verhältnis. Ein Wert um 1 deutet auf ein Gleichgewicht beider Seiten hin. Durchschnittliche Werte aus der deutschen Erwerbsbevölkerung liegen dagegen um 0,5 bis 0,6 (also eine deutliche Verschiebung des Quotienten Richtung Belohnungsfaktoren). Instrumente einer guten Weiterbildung wie Weiterbildungsverträge, feste Curricula mit geplanten Rotationen und regelmäßige Weiterbildungsgespräche waren mit einer geringeren psychosozialen Arbeitsbelastung assoziiert.

Als Fazit der Untersuchung stellen die Autoren fest, dass viele junge Ärztinnen und Ärzte die aktuellen Arbeits- und Weiterbildungsbe- dingungen als belastend empfinden, was nicht nur Konsequenzen für sie selbst, sondern auch für ihre Patienten haben kann. Es sollte im Interesse aller an der Organisation des Gesundheitswesens beteiligten Partner liegen, ein Umfeld herzustellen, in dem Ärztinnen und Ärzte dauerhaft gesund und effektiv arbeiten können. Nur so wird die wertvolle Ressource «Arzt» bestmöglich in der aktiven Patientenversorgung gehalten und eine hochwertige Patientenversorgung auch zukünftig gewährleistet.

Diesem Beitrag liegt die Studie Joachim SC, Bitzinger D, Arnold H, et al.: Die Arbeits- und Weiterbildungssituation junger Ärztinnen und Ärzte in Deutschland - eine zusammenfassende Analyse von Befragungsergebnissen aus sechs Fachrichtungen. Das Gesundheitswesen 2019;DOl:10.1055/a-0945-9387 zugrunde.

Aus dem Bündnis Junge Ärzte (BJÄ) waren folgende Nachwuchsgruppen an der Analyse beteiligt:

- Junge Internisten der Deutschen Gesellschaft für Innere Medizin e.V. (DGIM) und das Junge Forum des Berufsverbandes Deutscher Internisten e.V. (BDI)

- Wissenschaftlicher Nachwuchs (WAKWiN) und Junge Anästhesie der Deutschen Gesellschaft für Anästhesiologie und Intensivmedizin e.V. (DGAI) sowie der youngBDA des Berufsverbandes Deutscher Anästhesisten e.V. (BDA)

- German Society of Residents in Urology e.V. (GeSRU)

- Forum Junge Gynäkologie der Deutschen Gesellschaft für Gynäkologie und Geburtshilfe e.V. (DGGG)

- Arbeitsgemeinschaft Young DOG der Deutschen Ophthalmologischen Gesellschaft e.V. (DOG)

- Forum Junge Radiologie der Deutschen Röntgengesellschaft e.V. (DRG)
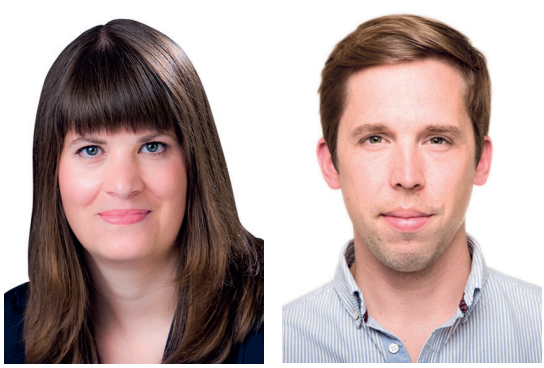

Die beiden ehemaligen Campus-Koordinatoren Prof Dr. Stephanie Joachim (OphthalmoCampus) und Dr. Matthias Raspe (PneumoCampus) waren an der Studie beteiligt.

Das Bündnis Junge Ärzte (BJÄ)

Das BJÄ ist der breite Zusammenschluss ärztlicher Nachwuchsgruppen in Deutschland. Das BJÄ vertritt die Belange junger Ärztinnen und Ärzte im Gesundheitswesen und bringt sich aktiv in aktuelle Themen ein - zum Wohle einer hochwertigen Patientenversorgung und guter ärztlicher Arbeits- und Weiterbildungsbedingungen.

Zur Internetseite der BJ $\ddot{A}:$ www.buendnisjungeaerzte.org.

Kontakt: Prof. Dr. Stephanie Joachim, Experimental Eye Research Institute, Universitäts-Augenklinik, Ruhr-Universität Bochum, In der Schornau 23-25, 44892 Bochum; Dr. Matthias Raspe, Medizinische Klinik mit Schwerpunkt Infektiologie und Pneumologie, Charité - Universitätsmedizin Berlin, Augustenburger Platz 1, 13353 Berlin, matthias.rapse@charite.de
30

Kompass Ophthalmol 2020;6:29-33

DOI: $10.1159 / 000505558$ 


\section{Aus der Praxis}

\section{Was wollen Chefärzte? Und wie passt das zu Deinen Erwartungen an die Weiterbildung?}

Wie stellst Du Dir den optimalen Arbeitgeber für die Facharzt-Weiterbildung vor? Und was wünscht sich Dein künftiger Chef? Im Dialog mit dem Publikum haben die Chefärzte Dr. Georg Hillebrand und PD Dr. Marko Fiege beim Operation Karriere-Kongress in Hamburg die gegenseitigen Erwartungen herausgearbeitet. Wir haben mit beiden gesprochen.

Ein weiß-rotes Absperrband teilt das Publikum in zwei Hälften: Die Medizinstudenten und jungen Ärzte, die beim Operation KarriereKongress in Hamburg im Hörsaal sitzen, dürfen bei diesem Vortrag nicht nur zuhören. Stattdessen können sie selbst aktiv werden und sich in verschiedene Situationen einfühlen. Die Zuhörer auf der einen Seite spielen die «Chefärzte»: Sie wollen für ihre Klinik möglichst geeignete Nachwuchskräfte rekrutieren. Auf der anderen Seite sitzen die "Weiterbildungsassistenten", die eine attraktive Stelle suchen, bei der sie viel für ihre Facharztweiterbildung lernen, aber bei der auch die Work-Life-Balance nicht zu kurz kommt.

Hillebrand und Fiege steuern die Diskussion mit Fragen: Welche Form sollte eine Bewerbung haben? Ist die Bewerbungsmappe noch zeitgemäß, oder reicht es, im Bewerbungsportal seine Unterlagen hochzuladen? «Eine E-Mail mit den Dokumenten als pdf-Datei im Anhang ist inzwischen üblich und meistens gleichwertig wie die gedruckte Bewerbungsmappe», erklärt Hillebrand, «allerdings sollten Sie keine Serienbriefe schreiben, sondern in der Bewerbung zeigen, dass Sie sich mit der Stelle auseinandergesetzt haben». «Wir stellen niemanden ein, der nicht zumindest bei uns hospitiert hat», ergänzt Fiege, «dann rede ich mit meinen Kollegen, ob der Kandidat ins Team passt. Besser ist es natürlich, wenn jemand schon im PJ bei uns war - dann wissen beide Seiten, was sie erwartet».

Was ist wichtig: Promotion? Auslandserfahrung? Ehrenamt? Eine Promotion sei nicht entscheidend - allerdings sei sie auch kein Nachteil, verrät Fiege weiter. Im besten Fall habe jemand in seiner Doktorarbeit schon bestimmte Fähigkeiten unter Beweis gestellt, die für die Weiterbildung wichtig seien.

Auslandserfahrung sei grundsätzlich gut; allerdings komme es darauf an, was genau jemand gemacht habe, erklärt Hillebrand: Wer zwei PJ-Tertiale auf Tahiti verbracht habe, müsse sich die Frage gefallen lassen, ob dabei nicht vielleicht die Freizeitgestaltung im Mittelpunkt gestanden habe. Etwas anderes sei ein Aufenthalt in einer Klinik für Tropendermatologie in Sri Lanka - das ist bei der Bewerbung von Vorteil. Gut sei es auf jeden Fall, wenn man soziales Engagement nachweisen könne - zum Beispiel in einer studentischen Initiative.

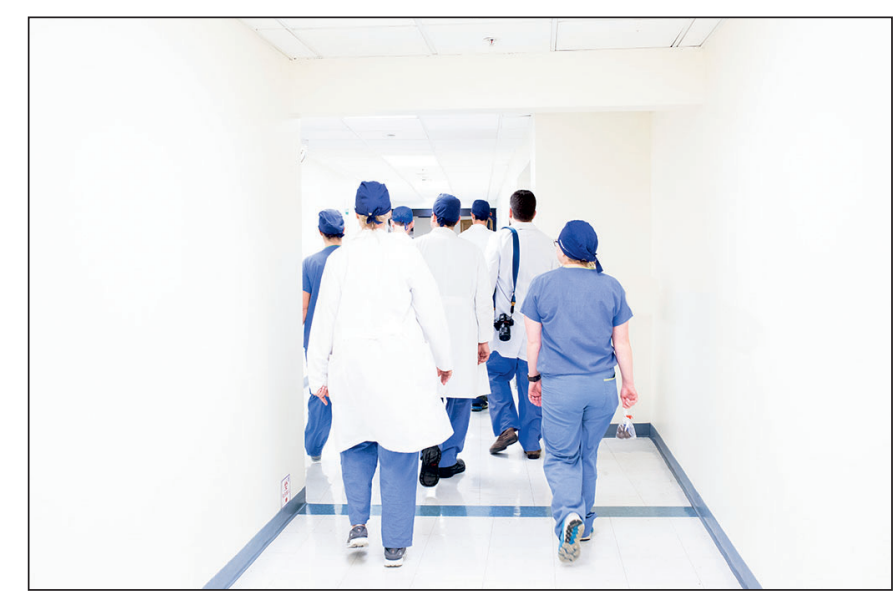

Bewerber müssen ins Team passen. Eine Hospitation ist oft der Schlüssel, das unter Beweis zu stellen. Foto von Luis Melendez auf Unsplash.

\section{Flexibilität ist angesagt}

Und was ist mit Lücken im Lebenslauf? «Das finde ich als Chefarzt oft extrem spannend, weil ich verstehen will, was jemand in der Zeit gemacht hat», verrät Fiege, «Das macht Menschen spannend, wenn sie im Leben auch mal über den medizinischen Tellerrand geguckt und etwas ganz anderes gemacht haben». Und auch Kinder sind grundsätzlich kein Problem - vor allem, weil die junge Ärztegeneration die Vereinbarkeit von Familie und Beruf immer mehr einfordere, erklärt Hillebrand. Wichtig sei es nur, keine völlig steifen Vorstellungen von der Arbeitszeit zu haben: «Wir versuchen, Eltern da entgegenzukommen. Aber ein großer Teil der Facharzt-Weiterbildung ist nunmal in Schichtdiensten organisiert. Da können sich einzelne nicht nur die Rosinen rauspicken - sonst wird es dem Rest des Teams gegenüber schnell ungerecht».

Für beide Chefärzte ist aber die Begeisterung für das Fach und die Arbeit als Arzt das allerwichtigste Kriterium, wenn es darum geht, einen neuen Kollegen einzustellen: «Das ist dieses Leuchten in den Augen», beschreibt Hillebrand, «Der Kandidat soll für das Fach brennen. Und wenn man das merkt, ist das schon eine gute Ausgangslage».

Originalquelle: «Was wollen Chefärzte? Und wie passt das du Deinen Erwartunden an die Weiterbildung?» Ein Beitrag auf www.operationkarriere.de (www.operation-karriere.de/karriereweg/assistenzart/waswollen-chefaerzte-und-wie-passt-das-zu-deinen-erwartungen-andie-weiterbildung.html) 


\section{Experten im Gespräch}

\section{Dr. Georg Hillebrand und PD Dr. Marko Fiege im Interview}

Im direkten Gespräch sind Dr. Hillebrand und PD Dr. Fiege noch weiter ins Detail gegangen.

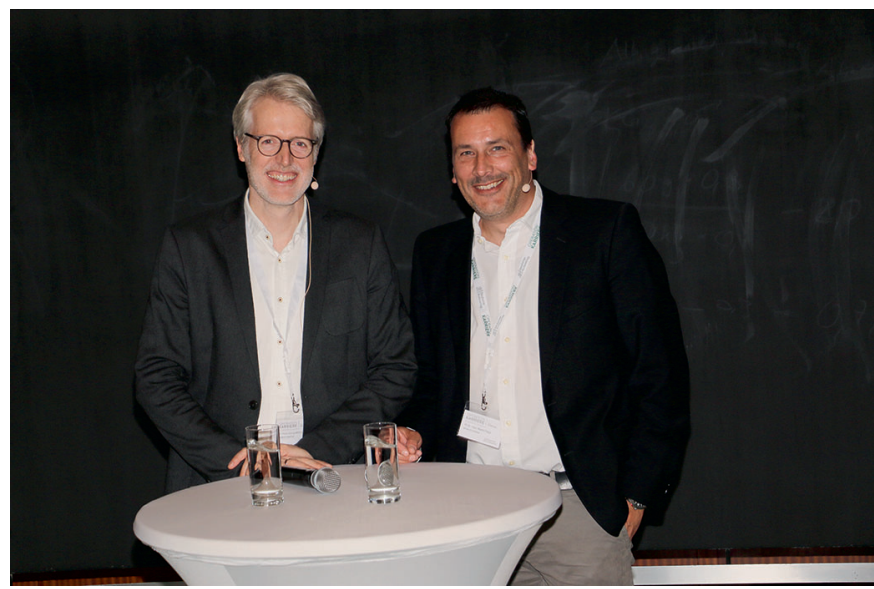

Dr. Georg Hillebran (links) ist Chefarzt der Klinik für Kinder- und Jugendmedizin am Klinikum Itzehoe. PD Dr. Marko Fiege (rechts) ist Chefarzt an der Klinik für Anäthesiologie am selben Klinikum. @ Hanke/Deutscher Ärzteverlag

Sehen Sie Diskrepanzen zwischen den Erwartungen der jungen Ärzte und den Erwartungen der Chefärzte?

PD Dr. Marko Fiege: Da muss es Diskrepanzen geben. Jemand, der eine Facharzt-Weiterbildung anfängt, hat ja ein ganz persönliches Interesse daran, in einer bestimmten Zeit gut ausgebildet zu werden und das auch mit seinem restlichen Leben gut kombinieren zu können - und das soll auch noch irgendwie nett sein. Das ist das Interesse der Weiterbildungsassistenten. Als Chefärzte haben wir natürlich auch ein Interesse daran, dass wir die besten Leute für unsere Klinik gewinnen und weiterbilden möchten - und es ist toll, wenn sie dann später erzählen, wie gut die Weiterbildung bei uns war. Aber wir haben natürlich auch ein Interesse an fleißigen Mitarbeitern, die für ihr Geld auch gute Arbeit machen - denn Weiterbildung ist nicht Ausbildung. Wir müssen die klinischen Belange abdecken und da ist es wichtig, dass die jungen Kollegen ab einem gewissen Zeitpunkt auch produktiv sind. Es ist immer herausfordernd, die Balance zwischen diesen beiden Interessen zu halten.

\section{Was können denn Kliniken in dem Zusammenhang tun, um für die jungen Ärzte attraktiv zu sein?}

Fiege: Ich glaube, man braucht ein gutes, strukturiertes Weiterbildungskonzept. Man muss eine Idee haben, wie man die Leute ausbildet: Was baut aufeinander auf und wann passt welcher Baustein gut ins Gesamtkonzept? Es gibt ja auch viele didaktische Gründe, warum eine bestimmte Reihenfolge bei den Inhalten sinnvoll ist. So ein Konzept muss es in der Klinik geben.

Dr. Georg Hillebrand: Es ist ja auch immer eine Frage, inwieweit sich Didaktik und der Anspruch an Lehre im Klinikalltag mit dem Team umsetzen lassen. Das eine ist eine Art Curriculum, in dem die Abläufe der Facharzt-Weiterbildung über fünf oder sechs Jahre struk- turiert sind. Das andere ist der Alltag: Inwieweit findet Lehre im Alltag statt? Das ist «Training on the job», weil natürlich in erster Linie die Patienten versorgt werden müssen. Manchmal ist die Ambulanz total voll - da muss schnell gearbeitet werden. Andererseits möchte der Assistenzarzt vielleicht jeden Fall sehr ausführlich nachbesprechen. Da muss man einen guten Kompromiss finden.

\section{Wie bringen Sie diese unterschiedlichen Erwartungen in der Praxis} unter einen Hut?

Hillebrand: Ich glaube, uns in der Kinderklinik in Itzehoe gelingt das ganz gut. Für uns im oberärztlichen und chefärztlichen Team ist Lehre ein wichtiger Teil unserer täglichen Arbeit. Wir machen jeden Morgen eine ausführliche Morgenbesprechung. Da besprechen wir alle neuen Patienten, bringen aber auch gleichzeitig noch einen Teaching-Aspekt mit rein. Da gibt es dann jeden Tag ein besonderes Thema, das kurz angesprochen wird. Außerdem gibt es jede Woche Fallkonferenzen, in denen wir reihum Fälle vorstellen. Und es gibt regelmäßige Besprechungen mit den Kollegen aus anderen Fachrichtungen. Ich glaube, das ist eine Herausforderung und ein Anspruch an das Team der leitenden Ärzte, dass sie sich neben der Patientenbetreuung und ihren administrativen Aufgaben nicht vergessen, dass die Ausbildung ein wesentlicher Teil des Jobs ist - und zwar qualitativ auf einem hohen Niveau. Und wenn man das berücksichtigt, spricht sich das rum - und das macht eine Klinik attraktiv für Bewerber.

Fiege: Ich glaube, dass es eine gewisse Ehrlichkeit in der curricularen Ausbildung geben muss. Für mich heißt das, die curricularen Vorgaben müssen realistisch zu dem passen, was wir auch umsetzen können. Wir betreiben zum Beispiel auch ein Simulationszentrum und machen simulationsgestützte Weiterbildung - aber im realen Leben geben die Patienten und der Klinikbetrieb vor, was passiert. Man kann in das Curriculum schreiben, dass jemand nach 92 Wochen eine bestimmte Behandlung durchführt. Aber dafür muss der passende Patient da sein und der Weiterbildungsassistent muss im Vorfeld die nötigen Grundlagen gelernt haben. Bei uns gibt es in der Weiterbildung Jahresthemen - da ist jemand dann schwerpunktmäßig zum Beispiel in der Intensivstation beschäftigt oder macht viel Notfallmedizin. Dieses grobe Raster halten wir auch seit längerer Zeit ziemlich dezidiert ein. Das lässt sich leichter umsetzen als ganz konkrete Pläne, was wann genau gemacht werden soll. Wenn man so ein grobes Raster hat, ist man schon relativ weit.

\section{Was geben Sie jungen Ärzten mit auf den Weg, damit der Einstieg in die Weiterbildung gut klappt?}

Hillebrand: Sie müssen für sich selbst eine Entscheidung treffen, dass es jetzt losgehen soll. Ich erwarte von niemandem, dass er seine künftige Karriere schon zu Beginn der Weiterbildung komplett vor Augen hat. Aber es sollte eine bewusste Entscheidung fallen: «Die nächsten zwei bis fünf Jahre bin ich jetzt in diesem Fach. Ich bin jetzt bereit zu starten und mein Leben darauf einzurichten.» Und dann klappt es. 
Fiege: Ich glaube, so eine Offenheit und Neugier für diesen Lebensabschnitt, der ganz anders ist als das Studium, ist wichtig: Das ist dann Real-Life und das heißt auch, man muss sich auf die Zusammenarbeit mit ganz unterschiedlichen Menschen aus unterschiedlichen Disziplinen einlassen. Da beginnt auf einmal auch ganz viel soziale Interaktion am Arbeitsplatz. In so einem Gefüge muss man sich erstmal zurechtfinden. Manchmal hilft es vielleicht, die eigenen Erwartungen ein Stück weit zurückzuschrauben - und die neuen Eindrücke auf sich wirken zu lassen. Die ersten Wochen und Monate sind - zumindest bei mir im Fach - extrem anstrengend. Das erzählen alle jungen Kollegen. Aber nach ungefähr einem halben Jahr ist auch wieder ein normales Leben außerhalb der Arbeit möglich.

\section{OPERATION \\ KARRIERE}

Originalquelle: Operation Karriere Hamburg, 14.06.2019, Impulsvortrag: «Neu in der Klinik - Erwartungen von Chefärzten und Weiterbildungsassistenten», PD Dr. med. Marko Fiege, Chefarzt der Klinik für Anästhesiologie und Dr. med Georg Hillebrand, Chefarzt der Klinik für Kinderund Jugendmedizin, beide Klinikum Itzehoe 